PSS PROCEEDINGS

\title{
Exclusive Diffractive Processes at HERA
}

\section{Armen Bunyatyan ${ }^{* \dagger}$}

DESY, Notkestrasse 85, 22603 Hamburg, Germany

E-mail: Armen. Bunyatyan@desy.de

The latest results on diffractive Vector Meson production and the Deeply Virtual Compton Scattering obtained by the H1 and ZEUS experiments at HERA are reviewed, with an emphasis on the transition from soft to hard interactions. The measurements are compared with several pQCD models.

35th International Conference of High Energy Physics

July 22-28, 2010

Paris, France

* Speaker.

†n behalf of the H1 and ZEUS Collaboration 


\section{Introduction}

Diffractive production of vector mesons (VM) and real photons $\gamma^{*} p \rightarrow V+Y$ (with $V=$ $\rho^{0}, \omega, \phi, J / \psi, \Psi^{\prime}, \Upsilon$ and $\left.\gamma\right)$, in which the proton may stay intact $(Y=p)$ or dissociate into a higher mass state $Y$ has been extensively studied at HERA. These processes span the whole range from "soft" diffraction, described by Regge phenomenology, to "hard" diffraction, where the hard scales, such as the heavy VM mass $\left(M_{V}\right)$ or the squared momentum transfer exchanges $Q^{2}, t$ at the respective photon and proton vertices (illustrated in Fig. 1) are involved and where the pQCD calculations can be applied. The VM production can be described in $\mathrm{PQCD}$ through the factorisation of virtual photon fluctuation into a $q \bar{q}$ colour dipole, diffractive dipole-proton scattering and $q \bar{q}$ recombination into the final state $\mathrm{VM}$ or photon. The interaction scale $\mu$ is given by the characteristic transverse size of the dipole, with $\mu^{2} \sim\left(Q^{2}+M_{V}^{2}\right)$. The dipole-proton scattering is modelled in $\mathrm{PQCD}$ as the exchange of a colour singlet two-gluon system or a BFKL ladder. Cross sections are thus given by the square of the gluon density in the proton. While the parton density distributions extracted from the inclusive scattering process do not contain information about correlations between partons, the exclusive VM production offers the possibility to learn more about these correlations, which are encoded in the generalised parton distributions (GPD). In particular the Deeply Virtual Compton scattering (DVCS, $\gamma^{*} p \rightarrow \gamma p$ ) process is ideal for this study since it has no com-

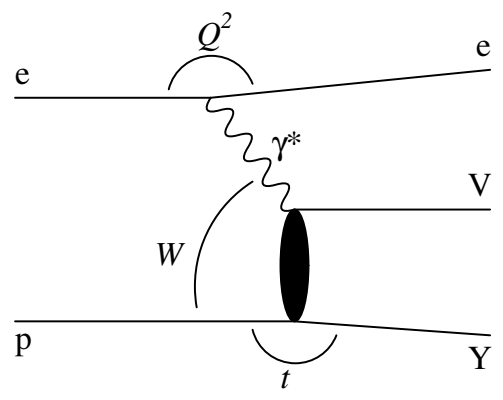

Figure 1: Generic diagram of diffractive vector meson electroproduction plications due to presence of the VM wave function in the final state description.

Among the most striking expectations in the transition from the soft to hard interactions is the change of the logarithmic derivative $\delta$ of the cross section with respect to the $\gamma^{*} p$ centre-of-mass energy $W$, from a value of about 0.2 in the soft regime to about 0.8 in the hard one and the decrease of the exponential slope $b$ of the differential cross section with respect to $t$, from a value of about $10 \mathrm{GeV}^{2}$ to an asymptotic value of about $5 \mathrm{GeV}^{2}$ when $\mu$ scale increases.

In this report a short review of the recent results of H1 and ZEUS experiments [1-7] is presented.

\section{From soft to hard interactions}

\subsection{Energy dependence of the cross sections}

The exclusive VM photoproduction cross sections $\sigma(\gamma p \rightarrow V p)$ are presented in the left side of Fig. 2 as a function of $W$. At high $W$, the cross section for all VMs follow an universal behaviour, $\sigma \sim W^{\delta}$. As the VM mass gets higher, a shallow $W$ dependence with $\delta \sim 0.2$ changes to a steeper one with $\delta \gtrsim 0.8$ (for $J / \psi, \Psi^{\prime}, \Upsilon$ ). The masses of heavy VMs provide the hard scale that allows the pQCD calculations to be performed. The exclusive $\Upsilon(1 S)$ photoproduction cross section [1] is reasonably well described by the different pQCD models (right side of Fig. 2).

The transition from soft to hard production of VM is studied further by varying the $Q^{2}$ scale. The cross sections $\sigma\left(\gamma^{*} p \rightarrow \rho^{0} p\right)$ and $\sigma(D V C S)$, presented in Fig. 3 as a function of $W$ for different 

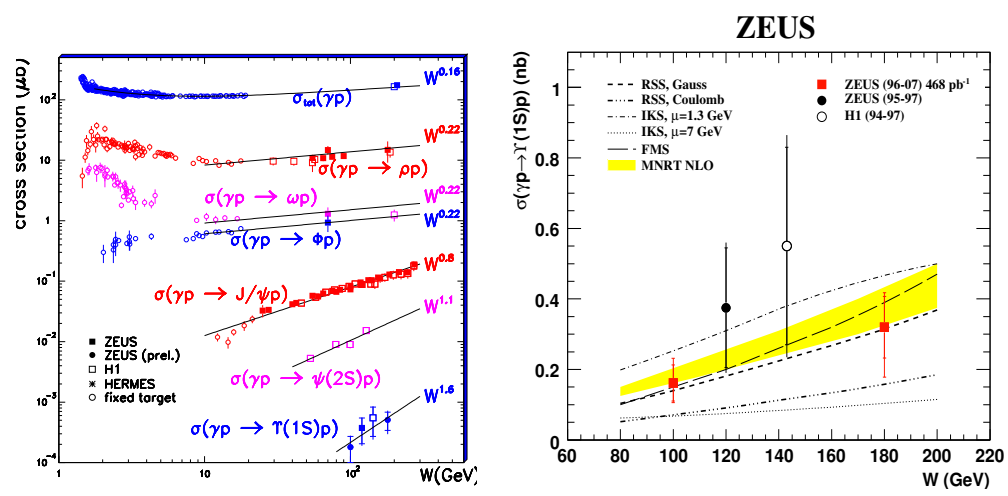

Figure 2: (left) The $W$ dependence of the cross section for diffractive VM production in the photoproduction $\left(Q^{2} \sim\right.$ 0 ), together with fits of the form $\sigma \sim W^{\delta}$. (right) The exclusive $\Upsilon(1 S)$ photoproduction cross section as a function of $W$. The lines denote predictions of the pQCD models.

intervals of $Q^{2}$, rise with $W$ for all $Q^{2}$ [2-4]. For $\rho$-mesons, this rise becomes steeper as the scale $Q^{2}$ increases, while for DVCS the $W$-dependence is rather steep even at lowest $Q^{2}$. Fig. 4 shows the result of the fits to the elastic VM electroproduction and DVCS cross section with a function $\sigma \sim W^{\delta}$ as a function of $\mu^{2}=Q^{2}+M_{V}^{2}$. All these measurements seem to line up a universal curve: $\delta$ increases as the scale becomes larger, in agreement with the expectations.
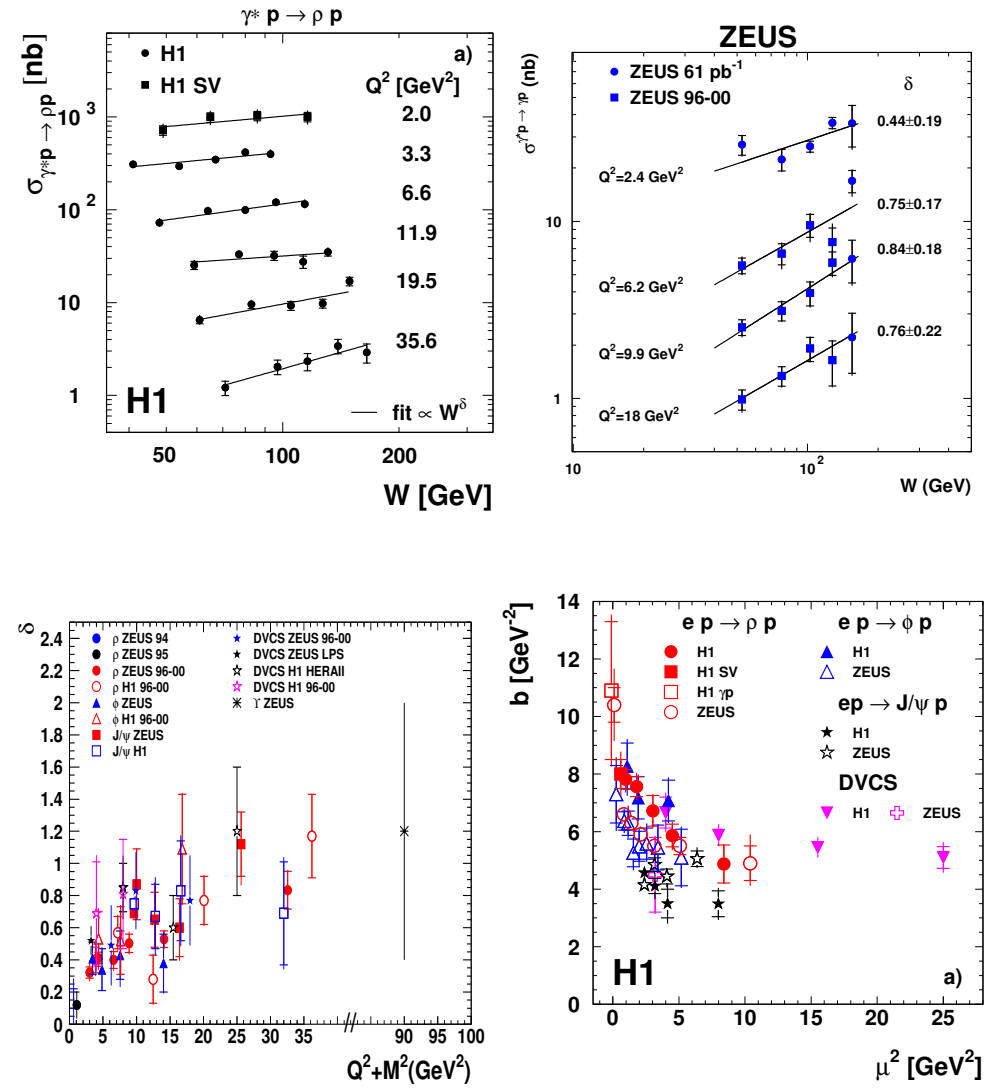

Figure 3: The $W$ dependence of exclusive $\rho$ electroproduction (left) and DVCS (right) for several $Q^{2}$ intervals.

Figure 4: (left) Compilation of the values of $\delta$ from a fit of the form $\sigma \sim W^{\delta}$ for exclusive $\mathrm{VM}$ production, as a function of $\mu^{2}=Q^{2}+M^{2}\left(\mu^{2}=Q^{2}\right.$ for DVCS). (right) Compilation of the values of $b$-slope from a fit of the form $d \sigma / d t \sim e^{b|t|}$ for exclusive VM production, as a function of $\mu^{2}$.

\section{$2.2 t$ dependence of the cross section}

The $t$ dependence of VM production cross section provides information on the transverse size and the dynamics of the processes, and on the scales relevant for the dominance of perturbative 
Figure 5: The $|t|$ and $W$ dependencies of the differential cross sections for the process $\gamma p \rightarrow J / \psi Y$. The lines show the predictions of several pQCD calculations.
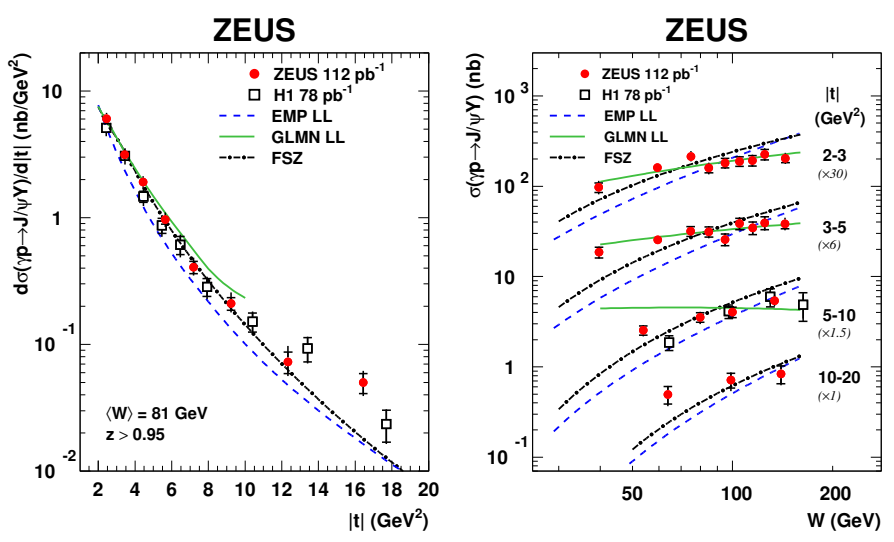

effects. For $|t| \lesssim 1-2 \mathrm{GeV}^{2}$, the $t$ distributions are exponentially falling as $d \sigma / d t \sim e^{-b|t|}$. The measured elastic $b$ slopes for the DVCS and VM production are shown in the right side of Fig. 4 as a function of the scale $Q^{2}+M_{V}^{2}$ [2-4]. With increasing of the scale, $b$ decreases to a universal value of about $5 \mathrm{GeV}^{-2}$.

\section{$2.3 J / \psi$ photoproduction at high $|t|$}

The diffractive photoproduction of $J / \psi$ at large $|t|$ is a two-scale process in which the mass of $J / \psi$ and $|t|$ define the hard scales at the photon and the proton vertices, respectively. In the region of relatively large $|t|>1 \mathrm{GeV}^{2}$ the dominant process of $J / \psi$ production is $\gamma p \rightarrow J / \psi Y$ where the proton dissociates into a low-mass nucleon state $Y$. At high $W$ this process is sensitive to BFKL dynamics [8]. In the region where the scale $|t|$ is smaller than the $J / \psi$ mass, the momenta of the gluon ladder are still expected to be ordered and thus the DGLAP approach is appropriate. As $|t|$ increases, the BFKL mechanism is expected to dominate. The differential cross sections $d \sigma / d|t|$ and $d \sigma / d W$ for this process are shown in Fig. 5 [5]. The DGLAP based model GLMN [9] gives a good description of the data up to about $|t|=5 \mathrm{GeV}^{2}$, but falls off slower than the data at higher $|t|$. We note, that none of the models describes the data over full $t$-range.

\section{Measurement of Pomeron trajectory}

The measurement of the $W$ dependence of $d \sigma / d t$ for fixed $t$ is used to extract the effective Pomeron trajectory $\alpha_{\mathbb{P}}(t)[6]$. The measured trajectory $\alpha_{\mathbb{P}}(t)$ for $\rho^{0}$ photoproduction, shown in Fig. 6, is fitted to a linear form to obtain the intercept $\alpha_{\mathbb{P}}(0)$ and the slope $\alpha_{\mathbb{P}}^{\prime}$. The combined fit to H1, ZEUS and Omega data leads to the values $\alpha_{\mathbb{P}}(0)=1.0871 \pm 0.0026$ (stat.) \pm 0.0030 (syst.) and $\alpha_{\mathbb{P}}^{\prime}=0.126 \pm 0.013$ (stat.) \pm 0.012 (syst.) $\mathrm{GeV}^{-2}$. The Pomeron intercept $\alpha_{\mathbb{P}}(0)$ is similar to the value expected for the Regge trajectory for hadron-hadron collisions, while the the slope $\alpha_{\mathbb{P}}^{\prime}$ is almost twice smaller than expected value of $0.25 \mathrm{GeV}^{-2}$ [10].

The variation of the Pomeron intercept $\alpha_{\mathbb{P}}(0)$ and slope $\alpha_{\mathbb{P}}^{\prime}$ with the scale $Q^{2}+M^{2}$ is studied for DVCS and elastic $\rho, \phi, J / \psi$ production. A common increase of $\alpha_{\mathbb{P}}(0)$ with scale is observed (Fig. 7), related to the hardening of the gluon distribution with the hard scale of the interaction. 


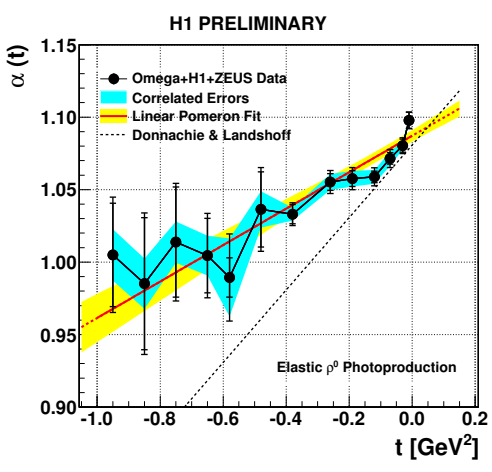

Figure 6: The effective Pomeron trajectory $\alpha_{\mathbb{P}}(t)$ measured from elastic $\rho^{0}$ photoproduction data of H1, ZEUS and Omega collaborations. Solid line shows the result from a linear fit of the form $\alpha_{\mathbb{P}}(t)=\alpha_{\mathbb{P}}(0)+\alpha_{\mathbb{P}}^{\prime}(t)$. The Pomeron trajectory $\alpha_{\mathbb{P}}(t)=$ $1.08+0.25 \cdot t[10]$ is shown as dashed line.
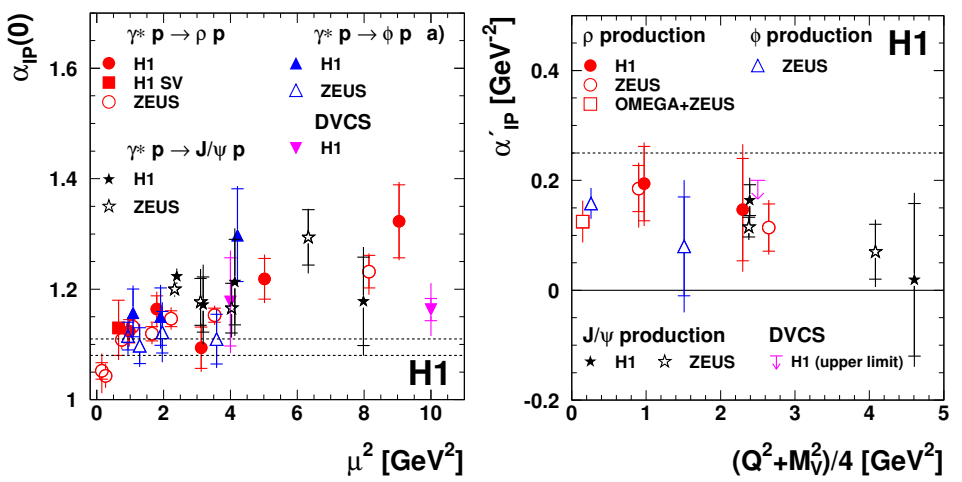

Figure 7: $\quad$ Evolution with the scale $\mu^{2}=\left(Q^{2}+M_{V}^{2}\right) / 4$ of the intercept $\alpha_{\mathbb{P}}(0)$ and slope $\alpha_{\mathbb{P}}^{\prime}$ of the effective Pomeron trajectory for elastic $\rho, \phi$, $J / \psi$ production and for DVCS $\left(\mu^{2}=Q^{2}\right.$ for DVCS $)$. The values $\alpha_{\mathbb{P}}(0)=1.08$ and 1.11 and $\alpha_{\mathbb{P}}^{\prime}=0.25 \mathrm{GeV}^{-2}$ typical for soft diffraction [10], are indicated by the dotted lines.

\section{4. $Q^{2}$ dependence of $\sigma_{L} / \sigma_{T}$ for $\gamma^{*} p \rightarrow \rho^{0} p$}

The ratio of VM production cross sections with transverse and longitudinally polarised photons, $R=\sigma_{L} / \sigma_{T}$, is sensitive to the interaction dynamics and the effects related to the interacting dipole size and the VM wave function. The ratio $R=\sigma_{L} / \sigma_{T}$ is extracted using the helicity analysis of the decay matrix elements [2]. $R$ measurements for $\rho, \phi$ and $J / \psi$ mesons are presented as a function of the scaling variable $Q^{2} / M_{V}^{2}$ in Fig. 8. A smooth and common behaviour is observed for the three VMs over the full energy range, from the fixed target experiments to the HERA collider measurements. The data are close to a law $R=Q^{2} / M_{V}^{2}$, represented by the dotted line, but they lie systematically below the line, with a slower increase of $R$ with increasing $Q^{2}$.

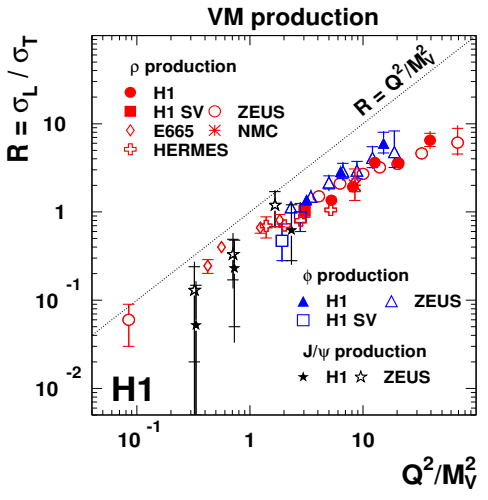

Figure 8: Ratio $R=\sigma_{L} / \sigma_{T}$ as a function of $Q^{2} / M_{V}^{2}$.

\section{Beam Charge Asymmetry (BCA) in DVCS}

Measurement of the BCA in DVCS gives a direct access to GPD in the proton. It is defined as $A_{C}(\phi)=\frac{d \sigma^{+} / d \phi-d \sigma^{-} / d \phi}{d \sigma^{+} / d \phi+d \sigma^{-} / d \phi}$ and presented in Fig. 9 [3]. Here $\phi$ is the angle between the plane formed by incoming and scattered electrons and that formed by the virtual photon and the scattered proton, defined in the proton rest frame. $d \sigma^{ \pm} / d \phi$ are the differential cross sections measured 
in $e^{ \pm} p$ collisions respectively. The fit to the measured points with a function $p_{1} \cdot \cos (\phi)$ is also displayed, with $p_{1}=0.16 \pm 0.04$ (stat.) \pm 0.06 (sys.). From the measurements of the BCA and of the DVCS cross section the ratio of the real to imaginary part of the DVCS amplitude is then derived, $\rho=0.20 \pm 0.05$ (stat.) \pm 0.08 (sys.). The GPD model [11] describes well the measurement within the experimental errors.

Figure 9: Beam charge asymmetry for DVCS process $\gamma^{*} p \rightarrow \gamma p$ as a function of $\phi$, measured in the range $0.05<|t|<1 \mathrm{GeV}^{2}$. The lines represents the result of a $p_{1} \cdot \cos (\phi)$ fit to the data points and the GPD model predictions.

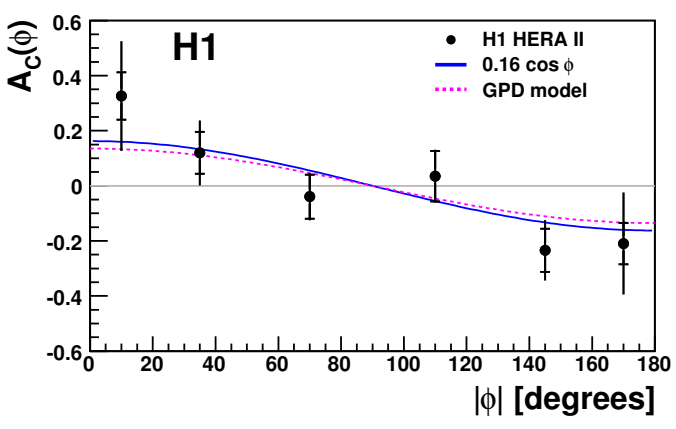

\section{Conclusions}

Presented here is a subjective selection taken from the wealth of new measurements of exclusive vector meson production processes delivered by $\mathrm{H} 1$ and ZEUS experiments at HERA collider. Much progress has been made over recent years, in the type of studies that can be performed, the precision achieved and in theoretical understanding. The high precision of the measurements can be used to refine the models and contribute to a better understanding of the vector meson wave functions and of the gluon density in the proton.

\section{References}

[1] S. Chekanov et al. [ZEUS Collaboration], Phys. Lett. B 680 (2009) 4 [arXiv:0903.4205 [hep-ex]].

[2] F. D. Aaron et al. [H1 Collaboration], JHEP 1005, 032 (2010) [arXiv:0910.5831 [hep-ex]].

[3] F. D. Aaron et al. [H1 Collaboration], Phys. Lett. B 681 (2009) 391 [arXiv:0907.5289 [hep-ex]].

[4] S. Chekanov et al. [ZEUS Collaboration], JHEP 0905 (2009) 108 [arXiv:0812.2517 [hep-ex]].

[5] S. Chekanov et al. [ZEUS Collaboration], JHEP 1005 (2010) 085 [arXiv:0910.1235 [hep-ex]].

[6] H1 Collaboration, "A measurement of the Pomeron trajectory based on elastic $\rho$ photoproduction" [H1prelim-09-016].

[7] ZEUS Collaboration, "Two pion diffractive electroproduction" [ZEUS-prelim-10-012].

[8] E. Kuraev, L. Lipatov and V. Fadin, Sov. Phys. JETP 44 (1976) 443.

[9] E. Gotsman et al., Phys. Lett. B 532 (2002) 37 [arXiv:hep-ph/0110256].

[10] A. Donnachie and P.V. Landshoff, Phys. Lett. B 296 (1992) 227.

[11] K. Kumericki, D. Mueller and K. Passek-Kumericki, Eur. Phys. J. C 58 (2008) 193 [arXiv:0805.0152 [hep-ph]]. 